\title{
Guided vortex motion in superconductors with a square antidot array
}

\author{
A. V. Silhanek, ${ }^{1}$ L. Van Look, ${ }^{1}$ S. Raedts, ${ }^{1}$ R. Jonckheere, ${ }^{2}$ and V. V. Moshchalkov ${ }^{1}$ \\ ${ }^{1}$ Nanoscale Superconductivity and Magnetism Group, Laboratory for Solid State Physics and Magnetism K. U. Leuven, \\ Celestijnenlaan 200 D, B-3001 Leuven, Belgium \\ ${ }^{2}$ Inter-University Micro-Electronics Center (Imec vzw), Kapeldreef 75, B-3001 Leuven, Belgium
}

(Received 7 February 2003; revised manuscript received 22 August 2003; published 10 December 2003)

\begin{abstract}
We have measured the in-plane anisotropy of the vortex mobility in a thin $\mathrm{Pb}$ film with a square array of antidots. The Lorentz force, acting on the vortices, was rotated by adding two perpendicular currents and keeping the amplitude of the net current constant. One set of voltage probes was used to detect the vortex motion. We show that the pinning landscape provided by the square antidot lattice influences the vortex motion in two different ways. First, the modulus of the vortex velocity becomes angular dependent with a lower mobility along the diagonals of the pinning array. Second, the vortex displacement is preferentially parallel to the principal axes of the underlying pinning lattice, giving rise to a misalignment between the vortex velocity and the applied Lorentz force. We show that this anisotropic vortex motion is temperature dependent and progressively fades out when approaching the normal state.
\end{abstract}

DOI: 10.1103/PhysRevB.68.214504 PACS number(s): 74.25.Fy, 74.78.Db, 74.25.Qt, 74.78.Na

\section{INTRODUCTION}

The interaction of a moving elastic medium with an array of obstacles has received much attention during the last years, in part due to a variety of condensed matter systems that can be described within this model. ${ }^{1-4}$ In general, the symmetry of the substrate over which the elastic medium moves, should also be reflected in the dynamic properties of the system. Particular interest has gone to the study of the flux line lattice (FLL) in superconductors with an artificial pinning potential, such as randomly distributed columnar tracks, ${ }^{5}$ arrays of submicron holes ("antidots") $)^{6-8}$ or magnetic dots. ${ }^{9,10}$

In a superconductor with a random distribution of pinning centers, an isotropic vortex mobility is expected with a vortex motion parallel to the Lorentz force. On the other hand, if the rotational symmetry of the pinning potential is broken, the response will be anisotropic, ${ }^{11}$ and guided vortex motion may appear.

Recent experiments ${ }^{12}$ have shown that the presence of twin boundaries in high temperature superconductors leads to a preferential motion of the FLL along the twinning planes. Guided vortex motion has also been found in systems with fourfold symmetry, such as square arrays of Josephson junctions. ${ }^{13}$ Theoretical work on vortex motion in a square array of pinning centers has forecasted guided vortex motion or channeling along the principal axes of the array, and along its diagonals. ${ }^{14-17}$ In these systems, the vortex velocity $\mathbf{v}$ preferentially snaps to the symmetry axes of the pinning array, leading to a misalignment between vortex velocity and the applied driving force $\mathbf{f}$. Even though this lock-in transition has been predicted in many numerical simulations for a square array of pinning centers, it had not yet been observed experimentally.

In this work, we report on electrotransport measurements on a $\mathrm{Pb}$ thin film with a square array of antidots, for the whole $360^{\circ}$ range of in-plane current orientations. This allows us to determine the misalignment between the applied current and the electric field generated by the vortex move- ment. We show clear evidence of guided vortex motion along the principal axes of the antidot array, and determine the trapping angle in the studied structure.

\section{EXPERIMENTAL ASPECTS}

The experiments were mainly performed in a 50-nm-thick $\mathrm{Pb}$ film (sample A), with a square antidot lattice (antidot size $\left.b^{2}=0.6 \times 0.6 \mu \mathrm{m}^{2}\right)$ of period $d=1.5 \mu \mathrm{m}$, which corresponds to the first matching field $H_{1}=\Phi_{0} / d^{2}=9.2 \mathrm{G}$, with $\Phi_{0}$ the superconducting flux quantum. We patterned the $\mathrm{Pb}$ film in a cross-shaped geometry (see Fig. 1) to allow electrical transport measurements at any in-plane orientation of the applied current. The cross consists of two $300 \mu \mathrm{m}$ wide strips containing the square array of antidots aligned with the principal axes of the cross.

This pattern was prepared by electron-beam lithography in a polymethyl metacrylate/methyl metacrylate (PMMA/ MMA) resist bilayer covering the $\mathrm{SiO}_{2}$ substrate. A $\mathrm{Ge}(20$ $\AA) / \mathrm{Pb}(500 \quad \AA) / \mathrm{Ge}(200 \quad \AA)$ film was then electron-beam evaporated onto this mask while keeping the substrate at 77 $\mathrm{K}$. Finally, the resist was removed in a liftoff procedure in

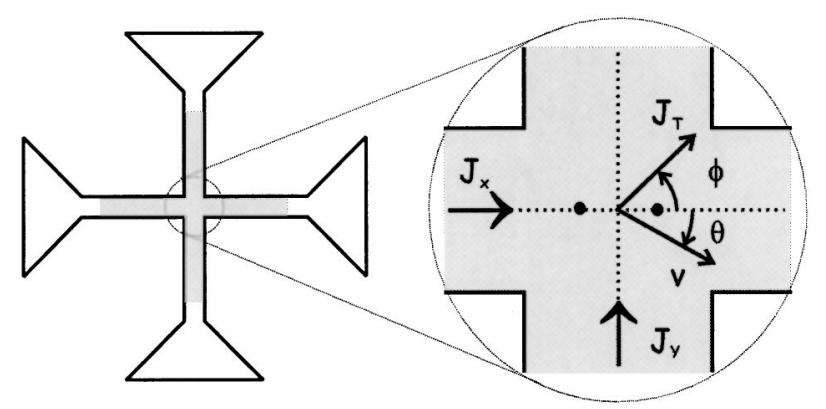

FIG. 1. Left: sketch of the cross-shaped $\mathrm{Pb}$ film with a square antidot array (gray area). Right: zoom in of the central part of the cross and orientation of the total applied current $\mathbf{J}_{T}$ and vortex velocity $\mathbf{v}$ with respect to the $\hat{\mathbf{x}}$ axis of the pinning array. The black dots represent the voltage probes. 
warm acetone. The sample has a $T_{c}=7.220 \mathrm{~K}$ with a transition width of $7 \mathrm{mK}$ for $H=0$.

The transport measurements were performed in a ${ }^{3} \mathrm{He}$ cryostat using two independent dc currents $J_{X}$ and $J_{Y}$, applied at the extremes of the cross legs, allowing to control the orientation $(\phi)$ of the total current $\mathbf{J}_{T}=J_{X}(\phi) \hat{\mathbf{x}}$ $+J_{Y}(\phi) \hat{\mathbf{y}}$, with respect to the principal axes of the square pinning array. These sources (Keithley M2400) are connected in series with a $1.2 \mathrm{k} \Omega$ resistor which provides an almost constant load for the source since its resistance is much larger than the resistance of contacts plus wires. The voltage contacts were aligned with the $\hat{\mathbf{x}}$ direction (see Fig. 1 , right panel) and located in the center of the cross with a separation $\sim 100 \mu \mathrm{m}$ in order to avoid possible current inhomogeneities. Each voltage contact has a spatial extent smaller than $30 \mu \mathrm{m}$. In the $V_{X}(\phi)$ measurements, we sweep the angle $\phi$ of the total current $\mathbf{J}_{T}$ while keeping its amplitude $J_{T}=\sqrt{J_{X}^{2}+J_{Y}^{2}}$ constant. The magnetic field $\mathbf{H}$ was applied perpendicular to the film surface.

It is worth to notice that in previous works, ${ }^{12,18}$ in order to determine the direction of the vortex movement, two sets of voltage probes arranged perpendicularly (aligned with each of the current directions), have been used. The simultaneous acquisition of the independent components of the electrical field $\mathbf{E}=\mathbf{v} \times \mathbf{H}$, allows one to determine the modulus and direction of the average vortex velocity $\mathbf{v}$ and therefore the misalignment between $\mathbf{v}$ and the applied Lorentz force $\mathbf{f}$ $=\mathbf{J}_{T} \times \boldsymbol{\Phi}_{\mathbf{0}}$. Although this procedure is strictly necessary for uniaxial systems, simple symmetry considerations associated with the periodic pinning landscape show that for higher degree of symmetry, it is possible to obtain the same information with a single pair of voltage contacts. Indeed, in our experiment, the intrinsic fourfold symmetry imposed by the pinning potential implies that $V_{Y}(\phi)=V_{X}\left(\phi+90^{\circ}\right)$, and therefore by measuring $V_{X}(\phi)$ in the whole angular range, we can deduce $V_{Y}(\phi)$.

\section{RESULTS AND DISCUSSION}

In Fig. 2(a) we show the field dependence of the resistance $R(H)$ for $J_{T}=3 \mathrm{kA} / \mathrm{cm}^{2}$ and $T=7.20 \mathrm{~K}$, for several angles $\phi$ of the total current. The most obvious features of this figure are the well defined dips at $\frac{1}{2} H_{1}$ and $H_{1}$, as a consequence of the reduction of the FLL mobility, reminiscence of the vortex configurations energetically stable in the equilibrium state. For low vortex density $\left(H<0.4 H_{1}\right)$, a linear field dependence characteristic of a flux flow regime $\rho_{\mathrm{FF}}=\rho_{n} H / H_{c 2}$, with $\rho_{n}$ the normal state resistivity and $H_{c 2}$ the upper critical field, is observed. ${ }^{19}$

It should be noted that, since the experiments were carried out very close to $T_{c}$, temperature stability plays a crucial role. For instance, the curve at $\phi=50^{\circ}$ in Fig. 2(a) exhibits a small asymmetry between the positive and negative branches of the magnetoresistance $[R(H) \neq R(-H)]$ because of a temperature drift of only $0.2 \mathrm{mK}$.

Due to the misalignment between the wire bounded voltage probes and the lithographically defined current contacts, the resistance in the normal state reaches the condition $R$
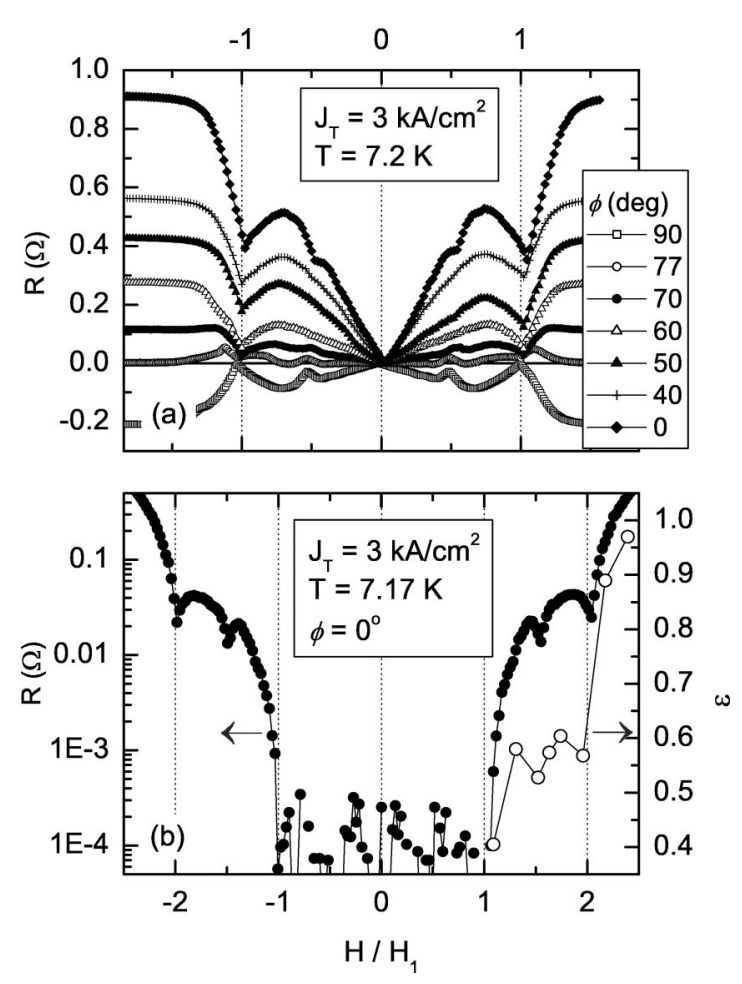

FIG. 2. Magnetoresistance $R(H)$ at $J_{T}=3 \mathrm{kA} / \mathrm{cm}^{2}$ for (a) several angles $\phi$ of the total current $\mathbf{J}_{T}$ at $T=7.20 \mathrm{~K}$ (b) $\phi=0^{\circ}$ and $T=7.17 \mathrm{~K}$. In (b), the field dependence of the eccentricity factor $\varepsilon=V\left(\phi=45^{\circ}\right) / V\left(\phi=0^{\circ}\right)$ is shown (open symbols).

$=0$ (i.e., $V_{X}=0$ ) at $\phi=77^{\circ}$ rather than at $\phi=90^{\circ}$. Remarkably, a finite resistance appears when penetrating in the superconducting phase $|H| \leqslant 15 \mathrm{G} \approx 1.6 H_{1}$ [open circles in Fig. 2(a) ]. Since the $R(H)$ curve at $\phi=77^{\circ}$ is symmetric around $H=0$, we rule out the possibility to attribute this dissipation to the Hall effect. We have also checked that thermoelectric voltages, generated by thermal gradients, have a negligible contribution to the total voltage.

Although a detailed analysis of the even transverse voltage (ETV) is not the purpose of this work, it is important to note that similar effects have been reported during the last years and today there is still no general consensus on the origin of this signal. In 1986, Glazman ${ }^{20}$ theoretically predicted the appearance of a transverse voltage as a consequence of the interaction between vortices and antivortices generated by the applied transport current when they penetrate the sample through displaced points along the current path. This effect manifests itself as a peak in the currentvoltage characteristic (CVC) near the critical current. According to this model, this effect should be stronger at zero field and progressively diminish as a biased applied field favors a vortex orientation. Since our measurements show that the detected transverse voltage persists even at very high fields, it is unlikely that the ETV observed in our samples arises from this effect.

Recently, Yamamoto and Ogawa $^{21}$ found ETV in $\mathrm{YBa}_{2} \mathrm{Cu}_{3} \mathrm{O}_{7}$ doped with $\mathrm{Ni}$ and $\mathrm{Zn}$, at high field. Strikingly the authors noticed that curves measured in samples produced and measured under same experimental conditions, give a different ETV response. They attribute this behavior 
to a nonuniform transport current at the superconducting transition. Since frequently the used samples do not have a geometry that is favorable for the six point measurements, this leads to an unknown current distribution. In such a case, it is convenient to determine the resistivity of the sample by means of the van der Pauw technique. These kind of measurements were first carried out by Francavilla et $a l^{22}$ and more recently by Vasek et $a .^{23}$ These authors show that the transverse voltage for all samples coincides qualitatively with the standard six point method used in our work thus indicating that inhomogeneous current distributions are not the source of the observed ETV.

In general, however, the ETV has been mostly discussed in terms of guided vortex motion. ${ }^{12,18,23-29}$ In this scenario, unlike the Glazman's theory where pinning is irrelevant, the pinning landscape plays a crucial role and the effect should be present even at high fields. All the experimental evidence reported so far (mainly on uniaxial pinning) shows that near the superconducting-normal transition a single (or multiple) anomalous peak in the transverse voltage develops. However, since we have observed that this effect persists in plain films without any orientational pinning, where transport properties should be isotropic, we can rule out the possibility to ascribe this ETV to guided vortex motion.

Similar anomalies have also been reported in the transverse voltage in Josephson junction arrays ${ }^{30}$ and in the longitudinal voltage in plain films. ${ }^{31}$ Although there is no general agreement about the origin of this effect, a possible explanation involves proximity effects due to the $N / S$ boundary in the voltage contacts. ${ }^{32}$ Clearly, a description of the origin of this ETV remains elusive at this point and deserves further experimental investigation.

We turn now to the longitudinal $\left(\phi=0^{\circ}\right)$ magnetoresistance measurements at lower temperatures. Figure 2(b) shows $R(H)$ for $\phi=0^{\circ}$ and $J_{T}=3 \mathrm{kA} / \mathrm{cm}^{2}$ at $T=7.17 \mathrm{~K}$. For fields $|H|<H_{1}$, where vortices are strongly pinned at the antidots, the dissipation lies below our experimental resolution. At this temperature, the maximum number $n_{s}$ of flux quanta that an antidot can hold is $n_{s} \approx b / 4 \xi(T) \sim 0.3 \ll 1,33$ where $\xi(T)$ is the superconducting coherence length. Under these circumstances, interstitial vortices appear in the sample for magnetic fields $H>H_{1}$. They are weakly pinned ("caged") at the interstitial positions between the repulsive saturated antidots. The matching features at $\frac{3}{2} H_{1}$ and $2 H_{1}$ are a result of their stable vortex configurations. In this scenario, where two species of vortices coexist, the motion of interstitial vortices should be strongly affected by the presence of those vortices which remain pinned. As field progressively increases, vortex-vortex interaction becomes more relevant in comparison with the vortex-pinning energy and eventually, close to the upper critical field $H_{c 2}$, a vortex liquid phase appears.

A more detailed description of the vortex dynamics can be obtained by analyzing the current-voltage characteristics for different fields. To that end, we have performed $I V$ measurements in a similar sample (sample B) having the same thickness and pinning array than sample A and using the standard four point measurements with the applied current parallel to the principal axis of the square array. ${ }^{34}$ Due to the small

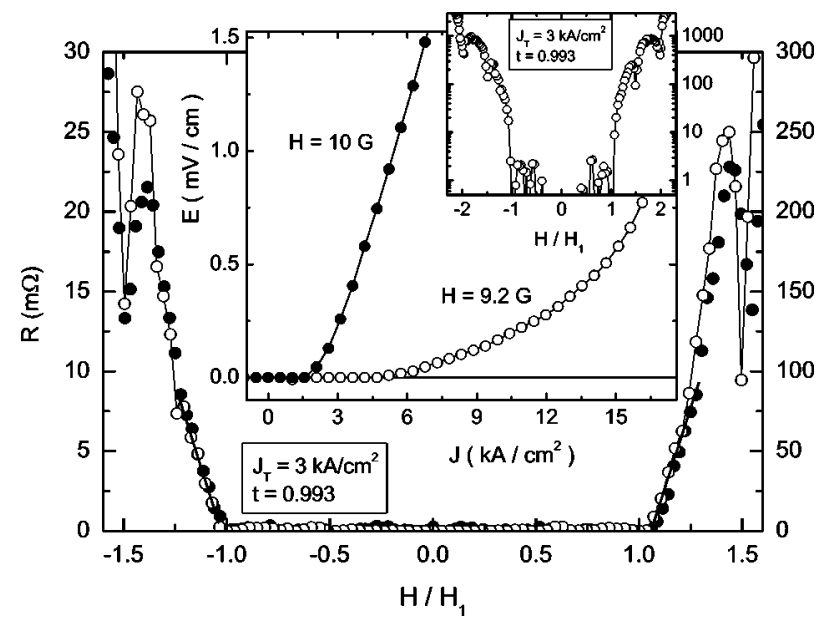

FIG. 3. Main panel: zoom in of the magnetoresistance data already shown in Fig. 2 (solid circles) together with the data for sample B (open circles) at the same current and reduced temperature. The straight lines indicate $R(H) \propto H-H_{1}$. The bigger inset shows the $E-J$ curves for sample B at $t=0.993$ for $H=9.2$ and 10 G. The upper inset shows the field dependence of the resistance $R(H)$ for $J_{T}=3 \mathrm{kA} / \mathrm{cm}^{2}$ at $t=0.993$ in sample B.

difference $(\sim 27 \mathrm{mK})$ in the value of the critical temperature between samples A and B, any comparison of the data should be performed at the same reduced temperature $t=T / T_{c}$. Most of the data presented in this work for both samples were recorded at $t=0.993$ corresponding to $T=7.170 \mathrm{~K}$ for sample A and $T=7.195 \mathrm{~K}$ for sample $\mathrm{B}$.

In the upper inset of Fig. 3 is shown the field dependence of the resistance $R(H)$ for sample B at $t=0.993$ and $J_{T}$ $=3 \mathrm{kA} / \mathrm{cm}^{2}$. As expected, the same features than those shown in Fig. 2 for the sample A are observed. This can be taken as a confidence test of the quality and reproducibility of the grown films and the nanoengineered pinning array. In the main panel a close up of this curve for $H \lesssim \frac{3}{2} H_{1}$ together with the data for the sample A (already presented in Fig. 2) is shown in linear scale.

In this figure we can see that for fields right above the first matching field, where interstitial caged vortices are easily channeled through the rows of flux lines pinned by the holes, $R \propto H-H_{1} \cdot{ }^{19}$ Since the dissipation is given by $V$ $=a n(H) v(H)$ [where $a$ is a constant, $n(H) \propto H-H_{1}$ is the number of flux lines crossing the voltage contacts and $v(H)$ the average vortex velocity], we can conclude that the average vortex velocity does not depend on field. This behavior can be understood within a scenario where a fraction 1 $-H / H_{1}$ of the total number of flux lines (interstitial) are in a flux flow motion channeled by the pinned vortices. According to this picture, in this regime a linear response should be observed in the $I V$ characteristics. In the main inset of Fig. 3 we show the $E-J$ curves ( $E$ is the electric field and $J$ the current density) for $H=H_{1}=9.2 \mathrm{G}$ and $H=10 \mathrm{G}$. We clearly observe that at $H=9.2 \mathrm{G}$ and for currents above the depining current $J_{\mathrm{dp}} \sim 5 \mathrm{kA} / \mathrm{cm}^{2}$ a nonlinear $E-J$ develops. In contrast, at $H=10 \mathrm{G}$ a linear $E-J$ relationship is observed for $J>J_{\mathrm{dp}} \sim 1.5 \mathrm{kA} / \mathrm{cm}^{2}$, in agreement with a flux flow regime. For higher fields, the interaction between interstitial 


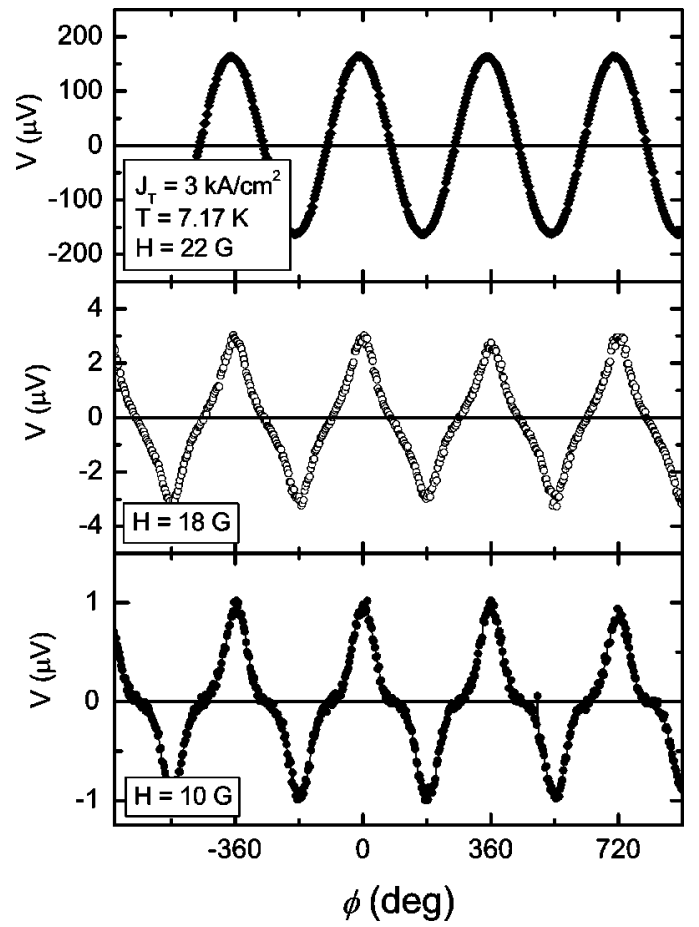

FIG. 4. Angular dependence of the voltage $V_{X}(\phi)$ for $J_{T}$ $=3 \mathrm{kA} / \mathrm{cm}^{2}$ and $T=7.17 \mathrm{~K}$, at $H=10,18$, and $22 \mathrm{G}$.

vortices becomes stronger and a deviation from linearity is observed.

Even though part of data corresponds to a linear regime, we mostly show data recorded in nonlinear regimes. In this case, the current path in the sample is nontrivial and the superposition of the two independent applied currents might lead to a net angle of the total current different from the desired angle. ${ }^{35}$ However, recent works in high-temperature superconductors ${ }^{12,18,27}$ have shown that although the signatures of a guided vortex motion develops in the liquid vortex state (where the response is ohmic), these features persist in the vortex solid phase. Furthermore, these authors noticed that the effect is progressive and without sharp changes at the Bose glass transition where the CVC are strongly nonlinear.

In order to study the role of the periodic pinning array on the vortex dynamics in each of these regimes, we have measured the in-plane angular dependence of the voltage $V_{X}(\phi)$ for the same conditions of Fig. $2(\mathrm{~b})\left(J_{T}=3 \mathrm{kA} / \mathrm{cm}^{2}\right.$ and $T$ $=7.17 \mathrm{~K})$ at $H=10,12,14,15,16,18,20$, and $22 \mathrm{G}$. For all these fields $J_{T}>J_{\mathrm{dp}}$ and therefore full current and field penetration is ensured. The curves $V_{X}(\phi)$ for some of them are presented in Fig. 4.

The upper panel of Fig. 4 shows $V_{X}(\phi)$ close to the normal state $\left[H_{c 2}(7.17 \mathrm{~K}) \approx 25 \mathrm{G}\right]$. The recorded signal follows a smooth sine function dependency characteristic of an isotropic regime with a constant vortex velocity independent of the current orientation. At $H=18 \mathrm{G}$, near to the second matching field, the response is quite different. Now, a sawtoothlike curve clearly manifests the existence of an in-plane anisotropy in the vortex movement. This anisotropy becomes even more evident as $H$ approaches to the first matching condition (lower panel). As we will show below, the charac-

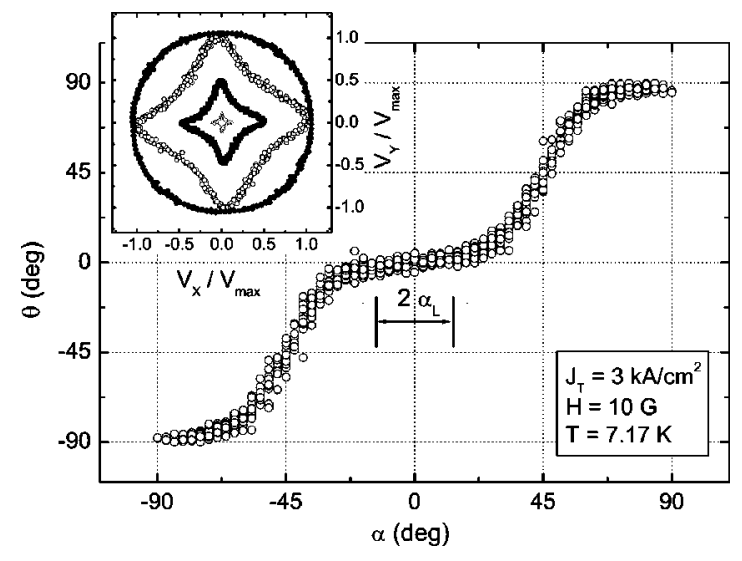

FIG. 5. Angle $\theta$ of the vortex velocity as a function of the Lorentz force orientation $\alpha$ for $J_{T}=3 \mathrm{kA} / \mathrm{cm}^{2}$ and $T=7.17 \mathrm{~K}$, at $H=10 \mathrm{G}$. Inset: polar graph $V_{X} \times V_{Y}$ for $J_{T}=3 \mathrm{kA} / \mathrm{cm}^{2}$ and $T$ $=7.17 \mathrm{~K}$, at (from inner to outer) $H=10,14,16$, and $22 \mathrm{G}$.

teristic shape of $V_{X}(\phi)$, alternating well pronounced peaks and plateaus, is a fingerprint of guided vortex motion.

As we pointed out previously, knowing the longitudinal component of the voltage $V_{X}(\phi)$, we can deduce the transversal component $V_{Y}(\phi)=V_{X}\left(\phi+90^{\circ}\right)$. This allows us to build a polar graph $V_{X} \times V_{Y}$, as shown in the inset of Fig. 5 for $H=10,14,16$, and $22 \mathrm{G}$, where the curves have been scaled for the sake of clarity. The circular shape of the outer curve $(H=22 \mathrm{G})$ reflects the rotational invariance of an isotropic conductor. As field decreases, the shape of the polar plot smoothly evolves from a circle, through a rhombus (not shown) and eventually data lies along an astroid for $|H|$ $<20 \mathrm{G}$, indicating that vortices move faster along the principal axes $[ \pm 1,0],[0, \pm 1]$ (easy direction) of the pinning structure than along the diagonals (hard direction).

The degree of anisotropy of the vortex velocity is given by the eccentricity factor $\varepsilon=V\left(\phi=45^{\circ}\right) / V\left(\phi=0^{\circ}\right)$. In case of a circular shape, $\varepsilon=1$, whereas for a rhombus $\varepsilon=1 / \sqrt{2}$. In Fig. 2(b) we show the anisotropy $\varepsilon$ from the analysis of the data at $\mathrm{T}=7.17 \mathrm{~K}$, as a function of field. We clearly observe that $\varepsilon(H)$ reproduces the behavior of the magnetoresistance $R(H)$. According to the theory, ${ }^{13}$ the vortex dynamics should become more anisotropic as $J_{T}$ decreases towards the depinning current $J_{\mathrm{dp}}$. On the other hand, at constant $J_{T}$, a higher $J_{\mathrm{dp}}$ yields a lower dissipation. Therefore, a stronger anisotropy should be detected where $R(H)$ has local minima, in agreement with our observations. A similar $\varepsilon(H)$ behavior, but somewhat less pronounced was found at higher temperatures for all fields $|H|<H_{c 2}$. This result indicates that the anisotropic vortex motion is present regardless of the dynamic regime.

Once the polar graph is built, it is possible to extract the angle of the electric field $\theta_{E}=A \tan \left(V_{Y} / V_{X}\right)$ and hence the angle of the vortex velocity $\theta=\theta_{E}+90^{\circ}$ as a function of the Lorentz force orientation $\alpha=\phi-90^{\circ}$. In the main panel of Fig. 5 we show $\theta(\alpha)$ so obtained for $H=10 \mathrm{G}$, folded to the fourth and first quadrant. ${ }^{36}$ For an isotropic conductor, $\theta$ $=\alpha$ and a straight line should be observed. In contrast, a highly nonlinear response is found.

Starting from $\alpha=0$ and rotating the current in counter- 
clockwise direction, i.e., increasing the angle $\phi$, we observe that for low values of $\alpha$, the force $\mathbf{f}$ lies ahead of $\mathbf{v}$, which remains close to the $[1,0]$ axis $\left(\theta \sim 0^{\circ}\right)$. The misalignment $|\alpha-\theta|$ reaches its maximum value at the lock-in angle $\alpha_{L}$ $\sim 15^{\circ}$, beyond which the vortex movement is no longer restricted to follow the $[1,0]$ direction and a fast increase in $\theta$ is observed. At the $[1,1]$ orientation, force and velocity are aligned due to the fourfold symmetry of the underlying pinning potential. Above $[1,1]$, the Lorentz force lags behind the velocity vector which rapidly approaches the $[0,1]$ axis. This is the central result of the paper which clearly indicates the role played by the periodic pinning potential in the vortex dynamics.

Recent analytical and numerical simulations ${ }^{14}$ for a moving FLL in a superconducting films with square pinning array show that minor lock-in phases should also be present at $\pm 45^{\circ}$ orientations. Further features might be present near the driving angles along the symmetry directions of the pinning array other than the principal axes. ${ }^{15}$ However, no evidence of such phases were found in our experiments. The absence of these regimes may be attributed to the relatively large size of the antidots. Indeed, since the pinning potential is averaged in the direction of motion, it is expected that the movement at $\pm 45^{\circ}$ (diagonals of the square antidots) be progressively washed out as the size of the antidots increases. Well defined channels will exist only if $d / 4 b>1$. In our experi- mental conditions $d / 4 b=0.625$ and thus channeling effects should be suppressed. This assumption is confirmed in the weakly coupled superconducting wire network limit by simulations for square Josephson Junction arrays by Marconi and Dominguez ${ }^{17}$ where no particular effects appear at $\pm 45^{\circ}$.

\section{CONCLUSION}

In summary, using dc-transport measurements we have demonstrated that the interaction of a moving flux line lattice with a periodic pinning potential, breaks the rotational symmetry and induces a guided vortex motion along the principal symmetry orientations of the pinning array. As a consequence, we found that for orientations different than the symmetry directions, the vortex velocity is not aligned with the Lorentz force. This effect vanishes close to the normal state where the vortex movement becomes insensitive to the pinning symmetry.

\section{ACKNOWLEDGMENTS}

We would like to thank Clecio C. de Souza Silva for helpful discussions. This work was supported by the ESF "Vortex" Program, the Belgian Interuniversity Attraction Poles (IUAP), the Flemish FWO, and Research Fund K.U. Leuven GOA/2004/02 programs.
${ }^{1}$ P. Martinoli, Phys. Rev. B 17, 1175 (1978).

${ }^{2}$ K. Runge and B. Pannetier, Europhys. Lett. 24, 737 (1993).

${ }^{3}$ A. van Blaaderen, R. Ruel, and P. Wiltzius, Nature (London) 385, 321 (1997).

${ }^{4}$ J. Hu and R.M. Westervelt, Phys. Rev. B 55, 771 (1997).

${ }^{5}$ D. Niebieskikwiat, A. Silhanek, L. Civale, G. Nieva, P. Levy, and L. Krusin-Elbaum, Phys. Rev. B 63, 144504 (2001).

${ }^{6}$ M. Baert, V.V. Metlushko, R. Jonckheere, V.V. Moshchalkov, and Y. Bruynseraede, Phys. Rev. Lett. 74, 3269 (1995).

${ }^{7}$ V.V. Moshchalkov, M. Baert, V.V. Metlushko, E. Rosseel, M.J. Van Bael, K. Temst, R. Jonckheere, and Y. Bruynseraede, Phys. Rev. B 54, 7385 (1996).

${ }^{8}$ V.V. Moshchalkov, M. Baert, V.V. Metlushko, E. Rosseel, M.J. Van Bael, K. Temst, Y. Bruynseraede, and R. Jonckheere, Phys. Rev. B 57, 3615 (1998).

${ }^{9}$ D.J. Morgan and J.B. Ketterson, Phys. Rev. Lett. 80, 3614 (1998).

${ }^{10}$ M.J. Van Bael, K. Temst, V.V. Moshchalkov, and Y. Bruynseraede, Phys. Rev. B 59, 14674 (1999).

${ }^{11}$ L. Van Look, B.Y. Zhu, R. Jonckheere, B.R. Zhao, Z.X. Zhao, and V.V. Moshchalkov, Phys. Rev. B 66, 214511 (2002).

${ }^{12}$ H. Pastoriza, S. Candia, and G. Nieva, Phys. Rev. Lett. 83, 1026 (1999)

${ }^{13}$ V.I. Marconi, S. Candia, P. Balenzuela, H. Pastoriza, D. Domínguez, and P. Martinoli, Phys. Rev. B 62, 4096 (2000).

${ }^{14}$ C.C. de Souza Silva and Gilson Carneiro, Phys. Rev. B 66, 054514 (2002).

${ }^{15}$ C. Reichhardt and F. Nori, Phys. Rev. Lett. 82, 414 (1999).

${ }^{16}$ Gilson Carneiro, Phys. Rev. B 62, R14661 (2000); 66, 054523 (2002).
${ }^{17}$ V.I. Marconi and D. Dominguez, Phys. Rev. B 63, 174509 (2001).

${ }^{18}$ Vicent Berseth, Ph.D. thesis, Ecole Politechnique Federale de Lausanne, Switzerland, 1999; G. D'Anna, V. Berseth, L. Forr, A. Erb, and E. Walker, Phys. Rev. B 61, 4215 (2000).

${ }^{19}$ E. Rosseel, M. Van Bael, M. Baert, R. Jonckheere, V.V. Moshchalkov, and Y. Bruynseraede, Phys. Rev. B 53, R2983 (1996).

${ }^{20}$ L.I. Glazman, Sov. J. Low Temp. Phys. 12, 389 (1986).

${ }^{21}$ Yukio Yamamoto and Keiichi Ogawa, Physica C 382, 80-84 (2002).

${ }^{22}$ T.L. Francavilla, E.J. Cukauskas, L.H. Allen, and P.R. Broussard, IEEE Trans. Appl. Supercond. 5, 1717 (1995).

${ }^{23}$ P. Vasek, Physica C 364, 194 (2001).

${ }^{24}$ F.A. Stass, A.K. Niessen, W.F. Druyvesteyn, and J.V. Suchtlen, Phys. Rev. Lett. 13, 293 (1964).

${ }^{25}$ A.A. Prodan, V.A. Shklovskij, V.V. Chabanenko, A.V. Bondarenko, M.A. Obolenskii, H. Szymcak, and S. Piechota, Physica C 302, 271 (1998).

${ }^{26}$ V.V. Chabanenko, A.A. Prodan, V.A. Shklovskij, A.V. Bondarenko, M.A. Obolenskii, H. Szymczak, and S. Piechota, Physica C 314, 133 (1999).

${ }^{27}$ M. Basset, G. Jakob, G. Wirth, and H. Adrian, Phys. Rev. B 64, 024525 (2001).

${ }^{28}$ J. Azoulay, J. Low Temp. Phys. 125, 205 (2001).

${ }^{29}$ A.K. Soroka and M. Huth, Low Temp. Phys. 28, 842 (2002).

${ }^{30}$ B.J. van Wees, H.S.J. van der Zant, and J.E. Mooij, Phys. Rev. B 35, R7291 (1987).

${ }^{31}$ Y.K. Kwong, K. Lin, M.S. Isaacson, and J.M. Parpia, Phys. Rev. Lett. 65, 2905 (1990); M. Park, M.S. Isaacson, and J.M. Parpia, ibid. 75, 3740 (1995). 
${ }^{32}$ M. Park, M.S. Isaacson, and J.M. Parpia, Phys. Rev. B 55, 9067 (1997).

${ }^{33}$ G.S. Mkrtchyan and V.V. Schmidt, Zh. Éksp. Teor. Fiz. 61, 367 (1971) [Sov. Phys. JETP 34, 195 (1972)]; H. Nordborg and V.M. Vinokur, Phys. Rev. B 62, 12408 (2000).

${ }^{34}$ In the sample B, the misalignement between the applied current and the voltage contacts was significantly reduced by introducing lithographically defined voltage probes.
${ }^{35}$ M. Pannetier, R.J. Wijngaarden, I. Floan, J. Rector, B. Dam, R. Griessen, P. Lahl, and R. Wördenweber, Phys. Rev. B 67, 212501 (2003).

${ }^{36}$ As we have shown previously, at this particular field $(H$ $=10 \mathrm{G}$ ) a linear CVC is observed and therefore the principle of superposition of currents remains valid. This ensures that the actual angle of the net current coincides with the desired angle. 